\title{
Rare presentation of Guillain-Barre syndrome with complete heart block and neuropathic chest pain mimicking acute coronary syndrome
}

\author{
A N Kuruppuarachchi ${ }^{1}, \mathrm{~K}$ T Sundaresan ${ }^{1}$, T Thivakaran ${ }^{2}$ \\ Ceylon Medical Journal 2018; 63: 149-150 \\ DOI: http://doi.org/10.4038/cmj.v63i3.8713
}

\section{Introduction}

Guillain-Barre syndrome (GBS) is an inflammatory disorder caused by the body's immune system damaging the peripheral nervous system. The most common type of Guillain-Barre syndrome is acute inflammatory demyelinating polyradiculoneuropathy (AIDP) presenting as muscular weakness with areflexia arising in the lower limbs and ascending gradually in more than $90 \%$ of patients [1].

Though autonomic involvement is known to occur, extreme parasympathetic over activity causing complete heart block is rare in the early course of the disease. Wani et al presented the first case report of acute inflammatory demyelinating polyradiculoneuropathy in a patient presenting with complete heart block and Stoke-Adams attacks [2]. Greenland conducted a retrospective study among 16 ICU admitted patients with Guillain-Barre syndrome with respiratory insufficiency [3]. Brady arrhythmias, including sinus arrest or atrioventricular block rhythms, and or tachyarrhythmia, supraventricular as well as ventricular, were observed in 13 patients. Two patients required pacemaker insertion because of recurrent asystolic episodes. But none of them developed any serious autonomic dysfunction in the early course of the disease.

Autonomic dysfunction can be due to either predominant sympathetic over activity or predominant parasympathetic over activity. Mixed dysfunction is also identified with generally poor outcome $[4,5]$. We report a rare case of an autonomic variant of Guillain-Barre syndrome that presented with complete heart block. The authors couldn't find any similar case reports of GuillainBarre syndrome with complete heart block apart from the case reported by Wanni et al [2].

\section{Case report}

A 56-year-old previously healthy farmer presented with gradual onset of left sided tightening chest pain which reached maximal intensity over 6 hours. Chest pain radiated from the back to the front of his chest. No radiation to arm or neck was reported. Pain was associated with sweating. He was a manual worker, non-smoker and occasional drinker. He had no history of preceding gastro-intestinal or upper respiratory tract illnesses.

He was in pain, pulse rate was 42 beats per minute, blood pressure was $130 / 80 \mathrm{mmHg}$. There were no murmurs and no features of acute left ventricular failure. Neurological examination was normal. There were no skin rashes or eschar. An ECG taken at the Emergency Department revealed complete heart block with complete absence of $\mathrm{AV}$ conduction with ventricular escape rate of 45 beats per minute. Considering the acute nature of his presentation an acute ischemic event was considered and stat doses of asprin, clopidogrel and statin were given in the Emergency Department. However, subsequent ECGs failed to demonstrate any dynamic changes suggestive of ischaemia.

Twelve hours following admission the chest pain had not resolved. He also complained of bilateral neuropathic lower limb pain and back pain. There was no weakness of limbs and reflexes were preserved. There was no bowel or bladder involvement. He was treated with gabapentin and monitored closely. Following day, he developed lower limb weakness and areflexia and weakness gradually ascended. We arrived at a diagnosis of autonomic variant of Guillain Barre syndrome. Intravenous immunoglobulin $0.4 \mathrm{mg} / \mathrm{kg}$ daily for five days was started and the patient was transferred to ICU.

The trans thoracic 2D echo was normal and troponin

'Department of Internal Medicine, Teaching Hospital Batticaloa, ${ }^{2}$ Department of Neurology, Teaching Hospital Batticaloa, Sri Lanka.

Correspondence: ANK, e-mail: <nadee86sha@gmail.com>. Received 26 May 2018 and revised version accepted 03 July 2018. 
I was negative. CRP was $5 \mathrm{mg} / \mathrm{l}, \mathrm{ESR}$ was $18 \mathrm{~mm} / 1^{\mathrm{st}} \mathrm{hr}$, full blood count showed mild neutrophil leukocytosis. Serum electrolytes including sodium, potassium, calcium and magnesium were normal. CSF analysis on day 10 of the illness showed elevated protein of $110 \mathrm{mg} / \mathrm{dl}$ with normal cell counts. Nerve conduction study performed after clinical recovery, showed patchy abnormalities of low nerve conduction velocity and prolonged $\mathrm{F}$ waves and abnormal sural nerve potentials, with intact and normal motor amplitude, compatible with acute inflammatory demyelinating polyradiculoneuropathy in recovery.

After treatment with immunoglobulin the weakness and neuropathic pain significantly improved and complete heart block reverted to sinus rhythm within first 24 hours of treatment. He recovered completely without any residual neurological deficits and he did not need atropine or a pace maker to maintain the heart rate.

\section{Discussion}

Autonomic dysfunction during the course of GuillainBarre syndrome is well recognized, but presenting with extreme parasympathetic activity such as complete heart block in the early course of the disease is rare. Knowledge of Guillain-Barre syndrome as a cause of complete heart block may help, arriving at an accurate diagnosis. Combined parasympathetic and sympathetic dysfunction is also a recognized manifestation of the disease. Our patient also had sweating and complete heart block which may indicate mixed dysfunction, but he had good outcome after early immunoglobulin treatment. This index case is important due to two reasons. This case emphasizes that Guillain-Barre syndrome should be considered in the differential diagnosis of patients presenting with acute chest symptoms and cardiac conduction blocks mimicking acute coronary syndrome. Early immunoglobulin administration may lead to full recovery of this life threatening autonomic dysfunction.

\section{Conclusion}

Complete heart block with acute chest pain is a rare presentation of the autonomic variant of Guillain-Barre syndrome. Early immunoglobulin treatment is lifesaving in severe autonomic dysfunction and may retard disease progression and hasten recovery. Temporary pacing may be required in hemodynamically unstable patients.

\section{Conflict of interest}

Authors declare that there are no conflicts of interest.

\section{Acknowledgements}

We thank Dr. Rajeewan Francis (Consultant Cardiologist, Teaching Hospital, Batticaloa) and Dr. M. Umakanth (Consultant Physician, Teaching Hospital, Batticaloa) for assisting us in the management of this patient.

\section{References}

1. Adams R, Victor M. Principles of Neurology. New York: McGraw-Hill, 1985, 968.

2. Wani B, Misra M, Shah M, Mufti S. Acute inflammatory demyelinating polyradiculoneuropathy presenting as complete heart block and Stoke-Adams attacks. Postgrad Med J 1989; 65(760): 103-4.

3. Greenland P. Arrhythmic complications in the GuillainBarre syndrome. Arch Intern Med 1980; 140(8): 1053-5.

4. Lichtenfeld P. Autonomic dysfunction in the Guillain-Barré syndrome. Am J Med 1971; 50(6): 772-780.

5. Tuck R, McLeod J. Autonomic dysfunction in GuillainBarré syndrome. J Neurol Neurosurg Psychiatry 1978; 44(11): 983-90. 